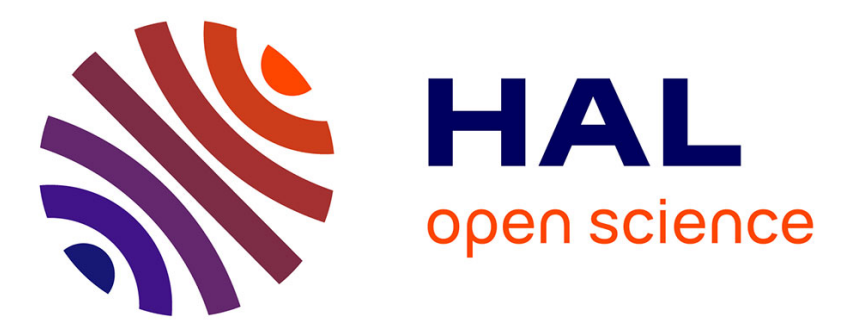

\title{
Effect of a high frequency magnetic field on plasma diffusion in a Q-machine
}

\author{
L.L. Artsimovitch, A.A. Ivanov, A.N. Lukjantschuk, V.L. Paperny, V.D. \\ Rusanov, S.S. Sobolev
}

\section{- To cite this version:}

L.L. Artsimovitch, A.A. Ivanov, A.N. Lukjantschuk, V.L. Paperny, V.D. Rusanov, et al.. Effect of a high frequency magnetic field on plasma diffusion in a Q-machine. Revue de Physique Appliquée, 1973, 8 (1), pp.49-52. 10.1051/rphysap:019730080104900 . jpa-00243653

\section{HAL Id: jpa-00243653 https://hal.science/jpa-00243653}

Submitted on 1 Jan 1973

HAL is a multi-disciplinary open access archive for the deposit and dissemination of scientific research documents, whether they are published or not. The documents may come from teaching and research institutions in France or abroad, or from public or private research centers.
L'archive ouverte pluridisciplinaire HAL, est destinée au dépôt et à la diffusion de documents scientifiques de niveau recherche, publiés ou non, émanant des établissements d'enseignement et de recherche français ou étrangers, des laboratoires publics ou privés. 


\title{
Classification \\ Physics abstracts 14.24 \\ EFFECT OF A HIGH FREQUENCY MAGNETIC FIELD ON PLASMA DIFFUSION IN A $Q$-MACHINE
}

\author{
L. L. ARTSIMOVITCH, A. A. IVANOV, A. N. LUKJANTSCHUK, V. L. PAPERNY \\ V. D. RUSANOV and S. S. SOBOLEV \\ I. V. Kurchatov Institute of Atomic Energy, Moscow, USSR
}

(Reçu le 14 janvier 1972)

\begin{abstract}
Résumé. - On présente des informations expérimentales sur la stabilisation des instabilités de dérive par un champ magnétique haute fréquence dans une machine $Q$. En régime d'instabilités de dérive dissipative la stabilisation provoque une baisse de l'intensité totale du spectre et diminue la diffusion. On compare la diffusion expérimentale avec la diffusion, prédite par la théorie pour le cas des oscillations de dérive amplifiées. On a observé une conformité satisfaisante entre l'expérience et la théorie.
\end{abstract}

\begin{abstract}
The paper presents experimental results on the interaction of a high frequency azimuthal magnetic field with drift and drift-dissipative oscillations in a $Q$-machine. Both the suppression of the oscillations and the decrease in the transverse diffusion in the regime of a driftdissipative instability are found.
\end{abstract}

It is known that $Q$-machine diffusion is of a « nonclassical $»$ character. Such an effect may be attributed both to development of instabilities in a plasma [1], [2] and to nonuniform heating of the hot end electrode [3], [4]. In the presence of intense oscillations the latter mechanism may be the determining factor. As has been shown by a number of authors, potential oscillations of different types may appear in the plasma depending on the $Q$-machine regime, in particular, on the density of the plasma charged particles and on the magnetic field. When the concentrations are not very high $\left(n \leqslant 5 \times 10^{9} \mathrm{~cm}^{-3}\right)$ and the magnetic fields are relatively weak, drift waves are excited in the plasma as described by Hendel and Politzer [5]. As the concentration increases and the electron-ion and ion-ion collisions play an important role the driftdissipative oscillations [5], [6] are excited in the plasma. The papers of Ivanov and others [7], [8] propose to stabilize the potential plasma oscillations by a high frequency magnetic field. The authors have observed suppression of the drift and drift-dissipative oscillations in a $Q$-machine by application of an azimuthal high-frequency magnetic field. The present paper develops these experiments with the purpose of revealing the effect of high frequency stabilization on the diffusion as well as to investigate in more detail the mechanism of interaction between a high frequency magnetic field and plasma oscillations.

The experiments were carried out with a potassium plasma in a single-ended $Q$-machine. Ionization took place on a hot tungsten electrode $3 \mathrm{~cm}$ in diameter. The plasma column was $100 \mathrm{~cm}$ long. The range of the concentrations was $2 \times 10^{8} \leqslant n \leqslant 2 \times 10^{10}$. The constant magnetic field was varied up to $4500 \mathrm{Oe}$. In contrast to the experiments already described the high frequency magnetic field was produced by a current of $70 \mathrm{kHz}$ flowing along four filaments arranged along the plasma column boundary. The current in adjacent filaments was oppositely directed, the amplitude reaching $45 \mathrm{~A}$ in each filament.

This method of producing a high frequency magnetic field is more convenient because the current carrying conductors are outside the plasma column and the magnetic field is applied more particularly to those regions of the plasma where the instabilities are maximum.

Radial distribution of the plasma concentration was measured by three double electric probes placed at distances of $20,40,60 \mathrm{~cm}$ from the heated end. The double electric probes were made from tungsten wire $0.2 \mathrm{~mm}$ in diameter. The collecting surface $5 \mathrm{~mm}$ long was oriented along the magnetic field lines so that the probe measured the transverse component of the electric field. The design of the probes allowed them to be heated before every serie of experiments preventing the potassium foil from settling on the collecting surface of the probe thus decreasing measurement errors. The oscillations were taken from the probe in the regime of floating potential and were fed to a differential amplifier (having a differentiality degree of about 100) and an input resistance of $100 \mathrm{M} \Omega$ to each channel. Then the signal was fed into a spectrum analyzer with a frequency resolution of $5 \mathrm{kHz}$. The averaged concentration was determined from the 
double probe saturation current by the conventional method.

Figures 1 and 2 show the radial distribution of the concentration in the absence of a H.F. magnetic field (curves I) and in the presence of a H.F. magnetic field (curves II) taken at a distance of $20 \mathrm{~cm}$ (Fig. 1) and $60 \mathrm{~cm}$ (Fig. 2) from the hot electrode. It is seen from the figures that as soon as the H.F. magnetic field is switched on the concentration increases and its

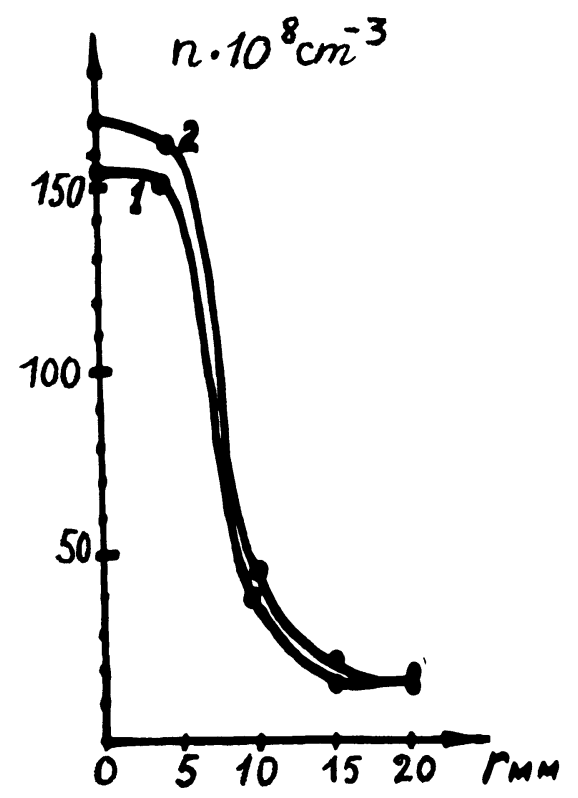

FIG. 1. - Radial distribution of the concentration without the presence of HF magnetic fields (curves I) and with the HF magnetic fields (curves II) taken at a distance of $20 \mathrm{~cm}$ from the hot electrode. $H_{0}=1.8 \mathrm{kOe}$.

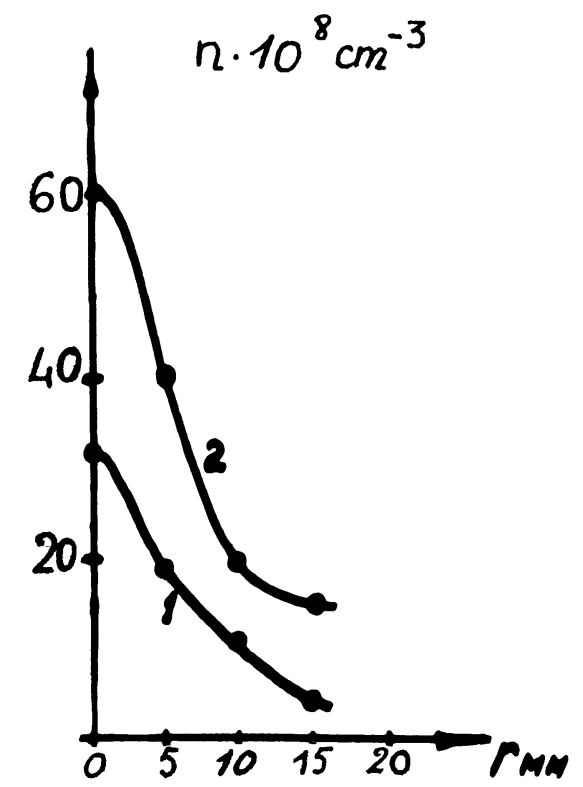

FIG. 2. - Radial distribution of the concentration without the presence of HF magnetic fields (curves I) and with the HF magnetic fields (curves II) taken at the distance of $60 \mathrm{~cm}$ from the hot electrode. $H_{0}=1.8 \mathrm{kOe}$. radial distribution becomes steeper. The concentration value near the hot end as well as the oscillation spectrum (Fig. 3) allow the oscillations appearing in this regime to be identified with drift-dissipative oscillations [5], [6] A numerical calculation using the following relation [9], [12]

$$
J_{\perp}(Z, r)=\sqrt{\frac{2 T_{\mathrm{i}}}{M_{\mathrm{i}}}} \frac{1}{r} \int_{0}^{r} \frac{\partial n(Z, r)}{\partial Z} r \mathrm{~d} r
$$

shows that the diffusion coefficient $\mathfrak{D}_{\perp}=j_{\perp} / \frac{\partial n}{\partial r}$ averaged over the plasma column length decreases from $300 \mathrm{~cm}^{2} / \mathrm{s}$ down to $100 \mathrm{~cm}^{2} / \mathrm{s}$. This value is only $2-3$ times greater than the classical one.

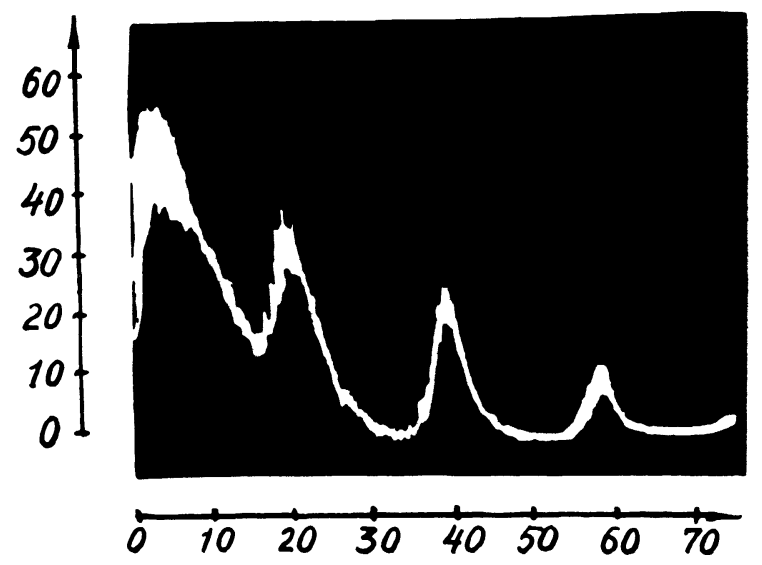

FIG. 3. - The potential oscillation spectra without the HF magnetic field $H_{0}=0.9 \mathrm{kOe}, n=1.5 \times 10^{10} \mathrm{~cm}^{-3}$.

Direct measurements of the transverse flux $j_{\perp}=\mathfrak{D}_{\perp} \frac{\partial n}{\partial r}$ were also carried out. The results obtained from these measurements are quantitatively consistent with the calculations using the equation for $j_{\perp}(Z, r)$. However, the interpretation of the results is rather difficult since $\tilde{j}_{\perp}$ depends both on the two quantities $\widetilde{\mathfrak{D}}_{\perp}$ and $\frac{\partial \tilde{}}{\partial r}$ varying with the HF magnetic field.

Therefore, $\widetilde{D}_{\perp}$ seems to be determined more reliably from the distribution $\tilde{n}(r)$ taking $v_{\mathrm{i}}$ constant with respect to the radius. This was proved by Motley et al. [12].

In a $Q$-machine it is likely that the diffusion should be influenced by plasma convection due to nonuniform heating of the hot end, inducing a centrifugal instability due to a radial electric field. To prevent nonuniform heating, special measures have been taken. It should be also noted that variation of $n(r)$ over the length (Fig. 1, 2) corresponds to the diffusion loss of the particles from the plasma column $\Delta x \sim \sqrt{\mathfrak{D} t}$ and not to the conventional losses $\Delta x \sim t v_{\text {conv. }}$. The rate of the diffusion expansion of the profile increases apparently with the noise power. 
To determine the role of $E_{r}$ a special experiment has been performed. Three tungsten cylinders coaxial to the plasma column were inserted into the chamber. The voltage relative to the hot end in the range of $-20,+20 \mathrm{~V}$ was applied to the cylinders. When the voltages $|V| \leqslant 3 \mathrm{~V}$ no variations in the concentration profiles were observed. Since the potential difference between the plasma and the hot end was less than 1-2 V, the effect of $E_{r}$ on the diffusion and centrifugal instability may be neglected. Taking into account the above considerations we think that the determining factor in the diffusion character should be the developed drift fluctuations.

Figures 3 and 4 show the effect of a high frequency magnetic field on the oscillation spectra for two different strengths of the constant magnetic field. In the absence of the HF magnetic field and when $H_{0}=0.9 \mathrm{kOe}$ a few oscillation modes are observed in the spectrum, while as $H_{0}$ increases $\left(H_{0}=1.8 \mathrm{kOe}\right)$ the spectrum becomes appreciably narrower within the region of low frequencies. This coincides with the experiments made earlier. Applica-

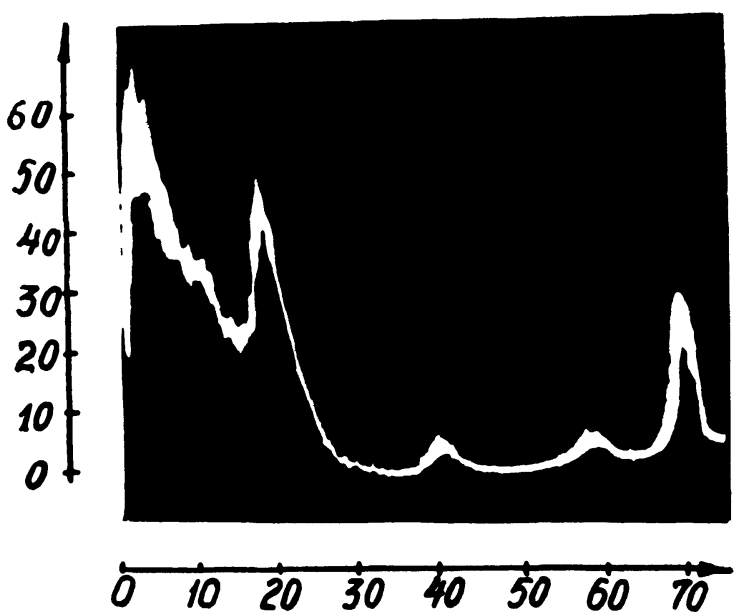

FIG. 4. - The potential oscillation spectra with $\mathrm{HF}$ magnetic field $H_{0}=0.9 \mathrm{kOe}, n=1.5 \times 10^{10} \mathrm{~cm}^{-3}$ 。
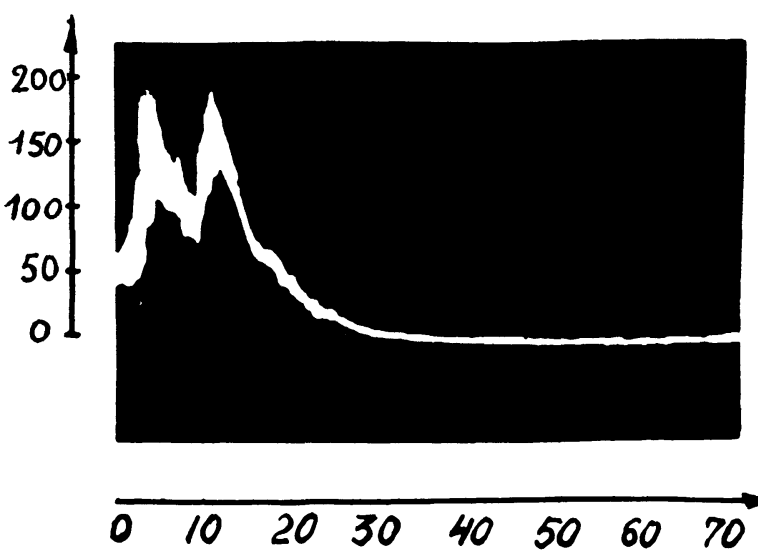

FIG. 5. - The potential oscillation spectra without the HF magnetic field $H_{0}=1.8 \mathrm{kOe}, n=1.5 \times 10^{10} \mathrm{~cm}^{-3}$. tion of a HF magnetic field gives rise to sharp decrease in integral noise density. As has been pointed out by Ivanov and others [10] the highest modes are stabilized better than the lower modes. Forced oscillations with the frequency of the HF magnetic field appear too. Together with these oscillations there are also lower frequency oscillations in the spectrum (in the range of a few kilohertz) free from influence of the HF magnetic field. This is associated with the fact that the wave vector component $K_{Z}$ of these oscillations along the magnetic field is large.

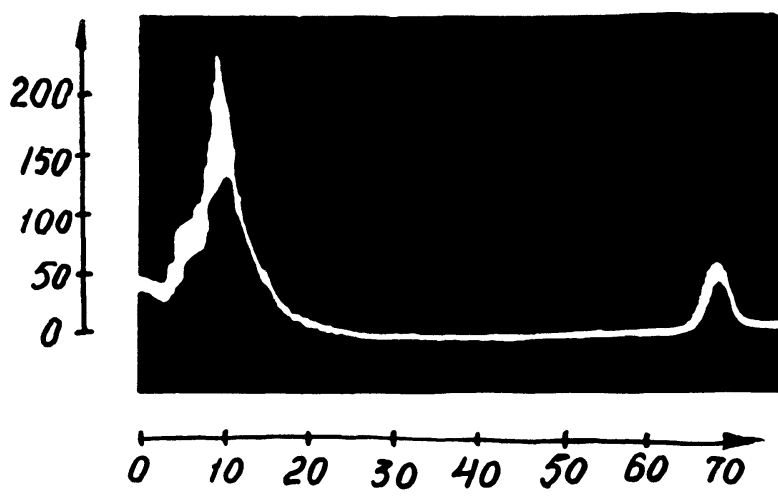

FIG. 6. - The potential oscillation spectra with HF magnetic field $H_{0}=1.8 \mathrm{kOe}, n=1.5 \times 10^{10} \mathrm{~cm}^{-3}$.

The decrease of the diffusion coefficient is associated with effective suppression of the drift-dissipative instability by a HF magnetic field. It can be estimated as follows

$$
\mathcal{D}_{\text {H.f. }} \sim \frac{\gamma_{\text {H.f. }}^{2}}{\Omega K_{\text {H.f. } \perp}^{2}} \sim \mathcal{D} \frac{\gamma_{\text {H.f. }}^{2}}{\gamma} \frac{\omega}{\Omega}\left(\frac{K_{\perp}}{K_{\text {H.f. } \perp}}\right)^{2}
$$

where $\mathscr{D}_{\text {H.f }}, \gamma_{\text {H.f., }}, K_{\text {H.f., }} \Omega$ - are respectively the diffusion coefficient, maximum increment of instability, transversal wave vector and oscillation frequency when a HF magnetic field is applied; $\mathfrak{D}, \gamma, K, \omega$ are the same quantities in the absence of a magnetic field. It has been shown in [11] that a HF magnetic field starts to influence the instability when the following relation is fulfilled :

$$
\beta=\frac{H_{1}^{2}}{H_{0}^{2}} \frac{K_{y} v_{\mathrm{Te}}}{\Omega v} \geqslant 1 .
$$

When the equality in the relation (2) is fulfilled the diffusion coefficient does not decrease and it equals $\mathcal{D}$, thus taking into account that

$$
\frac{\gamma_{\text {H.f. }}}{\gamma}=\frac{\omega}{\Omega \beta^{2 / 3}}
$$

we obtain from (1) :

$$
\mathfrak{D}_{\text {H.f. }} \sim \mathfrak{D}\left(\frac{\omega}{\Omega}\right)^{3}\left(\frac{K_{\perp}}{K_{\text {H.f. }}}\right)^{2}\left(\frac{H_{\mathrm{th}}}{H_{1}}\right)^{4 / 3}
$$


$H_{\text {th }}$ is determined from the condition $\beta=1$ (in the above formulae, $H_{0}$ is the strength of a constant magnetic field, $v_{\mathrm{Te}}$ is the thermal velocity, of electrons, $H_{1}$ is the strength of the high frequency magnetic field).

The quantity $H_{\mathrm{th}} / H_{1}$ was determined experimentally in this work. It is of the order of $\frac{1}{2}$ for the above operational conditions.

According to the evaluations made with the help of comparisons of oscillation amplitudes taken from single and double probes we obtained:

$$
\frac{K_{\perp}}{K_{\text {H.f. } \perp}} \sim 4, \quad \frac{\omega}{\Omega} \sim \frac{1}{4} .
$$

Therefore, it follows that $\left(\mathfrak{D}_{\text {H.f. }} / \mathscr{D}\right) \sim \frac{1}{8}$. Taking into account the approximate character of the evaluations this value is in satisfactory agreement with the experimental data.

Thus, it is shown that :

a) The diffusion due to the drift-dissipative oscillations is the principal mechanism of the particle losses from the plasma column.

b) The HF magnetic field suppresses effectively these oscillations and decreases the transverse diffusion coefficient almost to its classical value.

\section{References}

[1] Bernard M., Briffod G., Gregoire M., Proceedings of Conference of Physics of Quiescent Plasmas, Frascati, 1967 (c-1).

[2] Butchelnikova N. S. et al., PMTF 3 (1968) 89.

[3] Guilino E., Phys. Fluids 13 (1960) 7.

[4] Yassaby D. V., Mottley R. W., Phys. Fluids 1 (V12) (1969) 285.

[5] Hendel H. W., Politzer P. A., Proceedings of Conference of Physics of Quiescent Plasmas, Frascati, 1967 (B-7).

[6] Coppi B., Hendel H. W., Perkins F., Polotzer. Proceedings of Conference of Physics of Quiescent Plasmas, Frascati 1967 (B-8).
[7] Ivanov A. A., Rudakow L. Y., Teichmann Y. JETP 53 (1967) 1690.

[8] Ivanov A. A., Rudakow L. Y., Teichmann Y., JETP 54 (1968) 1580.

[9] Ivanov (A. A.), Rahimbabajev „Y. R., Rusanov V. D., JETP 52 (1967) 833.

[10] Ivanov A. A., Rasakov Y. B., LukJantschuk A. N., Rusanov V. D., Sobolev S. S., TeichmanN Y., JETP Letters 9 (1969) 356.

[11] Ivanov A. A., Teichmann Y., Czech J. Phys. B19 (1969) 914.

[12] Wong A. Y., Motley K. W., D’Angelo N., Phys. Rev. 133 (1964) 436.

[13] Chen F. F., Phys. Fluids 9 (1966) 2534 ; 10 (1967) 1647. 